\title{
Chemical Evolution of R-process Elements in the Hierarchical Galaxy Formation
}

\author{
Yutaka Komiya and Toshikazu Shigeyama \\ Research center for the early universe, University of Tokyo \\ Hongo, 7-3-1, Bunkyo-ku, Tokyo, 113-0033, Japan \\ email: komiya@resceu.s.u-tokyo.ac.jp
}

\begin{abstract}
The main astronomical source of r-process elements has not yet been identified. One plausible site is neutron star mergers (NSMs). From the perspective of Galactic chemical evolution, however, it has been pointed out that the NSM scenario is incompatible with observations. Recently, Tsujimoto \& Shigeyama (2014) pointed out that NSM ejecta can spread into much larger volume than ejecta from a supernova. We re-examine the chemical evolution of r-process elements under the NSM scenario considering this difference in propagation of the ejecta. We find that the NSM scenario can be compatible with the observed abundances of the Milky Way halo stars.
\end{abstract}

Keywords. stars: Population II, nuclear reactions, nucleosynthesis, abundances, Galaxy: evolution, Galaxy: halo

\section{Introduction}

The rapid neutron capture process (r-process) is thought to be a main process to synthesise elements heavier than the iron group in extremely metal-poor stars. But the dominant r-process element source in the universe is still matter of debate. Two possible sites have been proposed; core-collapse supernovae and neutron star mergers (NSMs).

From the viewpoint of chemical evolution, some difficulties of the NSM scenario have been pointed out (Argast et al. 2004). Since NSMs have a very low event rate and very large r-process yield per event, the NSM scenario predicts too large abundance scatter and many stars lacking r-process elements, in contradiction to observations (Roederer 2013, Komiya et al. 2014).

Recently, however, Tsujimoto \& Shigeyama (2014) pointed out that the ejecta from a NSM can spread into a very large volume since they have very large velocities of $10-30 \%$ of the speed of light. The stopping length, $l_{s}$, of ${ }^{153} \mathrm{Eu}$ at the speed of $0.2 c$ is estimated to be $\sim 2.6\left(n / 1 \mathrm{~cm}^{-3}\right)^{-1} \mathrm{kpc}$, where $n$ is the number density of neutral hydrogen.

In this paper, we revisit the chemical evolution of r-process elements considering this difference of propagation between NSM ejecta and supernova ejecta.

\section{The hierarchical chemical evolution model}

We use the chemical evolution model considering the hierarchical galaxy formation based on the extended Press-Schechter theory (Komiya et al. 2014, Komiya et al. 2015). The Galactic halo is formed through merger of proto-galaxies and the chemical abundance of each proto-galaxy evolves almost independently. In this work, we modify the chemical evolution model taking into account that NSM ejecta can go out from a host proto-galaxy and be captured by other proto-galaxies. 


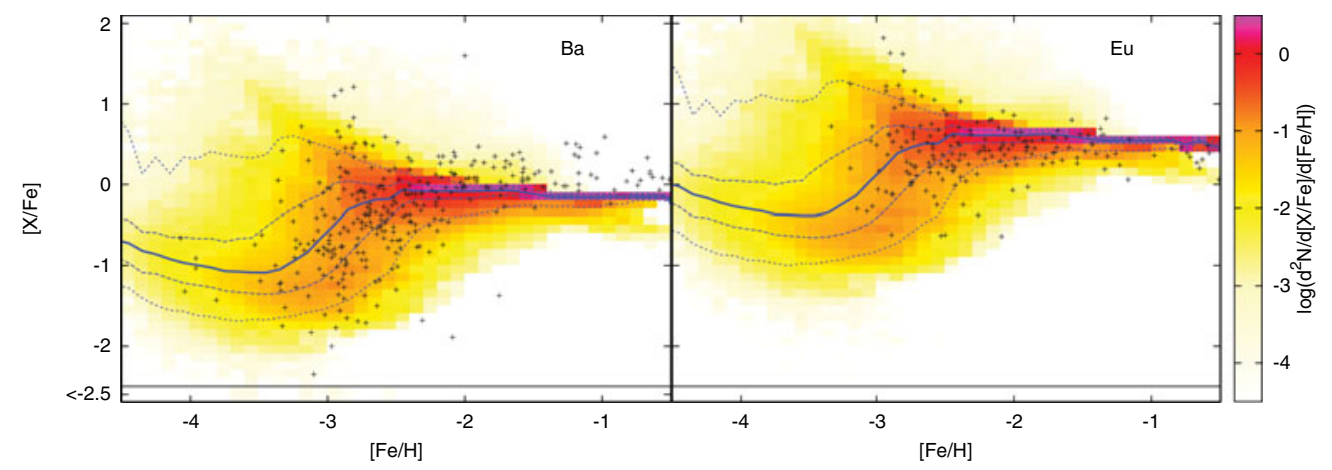

Figure 1. The predicted distribution of halo stars on the $[\mathrm{Fe} / \mathrm{H}]-[\mathrm{Ba} / \mathrm{Fe}]$ (left panel) and $[\mathrm{Fe} / \mathrm{H}]-[\mathrm{Eu} / \mathrm{Fe}]$ (right panel) planes is color coded. Black crosses are the observational sample from the SAGA database (Suda et al. 2008). Blue lines denote percentile curves for $5 \%$, $25 \%, 50 \%, 75 \%$ and $95 \%$ of the predicted distribution.

The escape fraction, $f_{\text {esc }}$, of NSM ejecta is assumed to be $f_{\text {esc }}=\exp \left(-\alpha R_{g, 0} / l_{s}\right)$, where $R_{g, 0}$ is the radius of the host proto-galaxy, and $\alpha$ is a free parameter. If r-process elements move straight, $\alpha=1$, but if it moves along a winding path due to a magnetic field, $\alpha>1$. We assume that the capture rate by other proto-galaxies is $f_{\text {cap }}=f_{\text {esc }}\left(R_{g, n}^{2} / 4 \pi d^{2}\right)\{1-$ $\left.\exp \left(-\alpha R_{g, n} / l_{s}\right)\right\}$, where $d$ is the distance between galaxies and $R_{g, n}$ is the radius of a capturing galaxy. We set the NSM event rate to be 1/1000 times the SN event rate and the coalescence timescale is $10^{6}-10^{8} \mathrm{yr}$ (Belczynski et al. 2006).

\section{Results and Discussion}

When we do not consider the escape of NSM ejecta from the host proto-galaxies, the majority of the stars with $[\mathrm{Fe} / \mathrm{H}]<-3$ show very low r-process abundance with $[\mathrm{Ba} / \mathrm{Fe}]$ $<-2.5$. On the other hand, if NSM ejecta directly go away from the proto-galaxy $(\alpha=1)$, most of the NSM ejecta go to the intergalactic space from small proto-galaxies.

We show the results of the model assuming $l_{s} / \alpha=4 \mathrm{kpc}$ in Figure 1 . In this case, a small fraction of NSM ejecta escapes from its host proto-galaxy and pollutes other proto-galaxies. This model well reproduces the observations of halo stars, as shown.

This result indicates that the NSM scenario is not rejected by chemical evolution, when we consider the propagation of NSM ejecta beyond the proto-galaxies.

\section{References}

Argast, D., Samland, M, Thielemann, F.-K., \& Qian, Y.-Z. 2004, A\&A, 416, 997

Belczynski, K., Perna, R., Bulik, T., et al. 2006, ApJ, 648, 1110

Komiya, Y., Yamada, S., Suda, T., \& Fujimoto, M. Y. 2014, ApJ, 783, 132

Komiya, Y., Suda, T., \& Fujimoto, M. Y. 2015, ApJ Letters, 808, L47

Tsujimoto, T., \& Shigeyama, T. 2014, A\&SA, 565, L5

Roederer, I. U. 2013, $A J, 145,26$

Suda, T., Katsuta, Y., Yamada, S., et al. 2008, PASJ, 60, 1159 